\title{
SIMPLIFIED PERIOD ESTIMATION OF ITALIAN RC BRIDGES FOR LARGE-SCALE SEISMIC ASSESSMENT
}

\author{
C. Zelaschi ${ }^{1}$, R. Monteiro ${ }^{1}$, and R. Pinho ${ }^{2}$ \\ ${ }^{1}$ Institute for Advanced Study of Pavia (IUSS) \\ Palazzo del Broletto, Piazza della Vittoria n.15, 27100 Pavia (Italy) \\ e-mail: \{claudia.zelaschi,ricardo.monteiro\} @iusspavia.it \\ ${ }^{2}$ University of Pavia, Department of Civil Engineering and Architecture \\ Via Ferrata n. 1, 27100 Pavia (Italy) \\ e-mail: rui.pinho@unipv.it
}

Keywords: Seismic assessment, Latin Hypercube sampling, RC bridges, Period estimation

\begin{abstract}
The nodes of a road transportation network of a certain region can represent a population of bridges. In view of a sound characterization of corresponding fragility or vulnerability functions, the knowledge of real data, such as geometrical and material properties available from a national bridge inventory database, is a crucial element. In fact, the outcome of damage seismic assessment can be strongly affected by the variability of such information. When only limited information for each node is known and the relevant structural behaviour needs to be assessed for e.g. loss estimation studies at macro-scale level, the use of statistical tools is of extreme importance. Accordingly, the present paper furnishes a double-fold contribution: (1) provide the systematic statistical characterization of geometrical and material properties of typical Italian reinforced concrete $(R C)$ bridges and (2) simplified formulae for the quick evaluation of the fundamental elastic period of vibration of $R C$ bridges. This allows the development of typological fragility curves for RC bridge classes, which might be chosen according to their fundamental period of vibration. As such, the bridges can be identified with a specific bridge class, characterized by a representative fragility curve, used for expedite large-scale seismic assessment.
\end{abstract}




\section{INTRODUCTION}

Severe past earthquakes had a significant impact on road network systems as well as considerable catastrophic effects on human life, buildings and economy. Examples of such disastrous events are the San Fernando earthquake in 1971, Loma Prieta in 1989, Northridge in 1994 and more recent events such as Italian and New Zealand earthquakes in 2009 and 2011, respectively. It is due to such consequences that the management of the emergency phase in the immediate aftermath of an earthquake is significant. One way to efficiently tackle such an issue is to rapidly assess the status of the main network components, which may exhibit weak seismic response, which, in turn, may cause infrastructure or cascading system disruptions. The main components of a network can be point-site or distributed components [1]. Bridges belong to the former category and they play an important role to ensure the efficiency of the network system itself and the interconnection between cities' strategic facilities or of its surrounding area [2]. A number of past studies have addressed the seismic assessment of individual bridge structures through nonlinear static or dynamic analysis [3-7]. However, such research was built upon the knowledge and the applications initially developed for building structures [8-12] and it resulted in the proposal of fragility curves for individual structures [13-16].

The derivation of fragility curves from a number of relevant geometrical or structural parameters taken from real case studies is of utmost importance for the accuracy of loss assessment investigations as part of decision-making strategies [17-18]. In such way, civil protection or other expert bodies can rely on them during the management of post-extreme event emergency phases. Yet, especially when dealing with broad areas, the development of fragility curves for each individual bridge can be unfeasible, mainly for the computational onus that such approach requires. Therefore, a parametric statistical approach may be particularly advantageous to characterize the parameters that affect the existing $\mathrm{RC}$ bridges response [19]. Given such characterization, one can simulate reliable data to represent additional structural configurations to fully characterize a wide range of bridges.

With the above in mind, the present study uses statistical tools to characterize populations of Italian bridges, targeting a specific typology. The collected data includes construction year, pier heights, total bridge length, span length, number of spans, superstructure area, pier main section sizes, compressive strength, tensile strength, shear strength, Young modulus and shear modulus of materials. In particular, geometrical and material properties associated to a real bridge stock are presented in a statistically usable form. Specifically, in the initial phase, they are summarized in terms of average (mean values) and corresponding dispersion (standard deviation and coefficient of variation). Successively, statistical distributions are assigned to each parameter by means of goodness-of-fit tests for small and larger samples. Such summary statistics are then used as input for the generation of a population of a specific bridge typology, representing a typical class of Italian bridges, through standard or Latin Hypercube-based Monte Carlo sampling schemes [20] in order to obtain a wide variety of bridge configurations. Finally, the configurations are analysed in order to define a simplified relationship between the period of vibration and the bridge geometrical properties that can be easily obtained or estimated through field survey or other equivalent methods. This relationship is intended for use in risk and loss assessment models and constitutes a first step towards the assignment and/or the generation of analytical fragility and vulnerability functions for specific bridge categories. In spite of its drawbacks, the relationship can be used as a potential proxy for seismic structural demand estimate of well-defined bridge typologies. It would also enable the possibility of rapidly sub-dividing bridge populations in classes for the expedite application of recent record selection procedures (e.g. conditional spectrum method) or early loss assessment 
approximations, through immediate assignment of a proper fragility curve for the assessment of damage level. Ultimately, this relationship may also be applied to estimate structural properties or damage levels when no detailed information on the bridge is available.

\section{SEISMIC ASSESSMENT FRAMEWORK}

The global framework involving the present study targets the development of typological fragility and vulnerability functions for specific bridge classes. The framework, illustrated in Figure 1, starts with the random generation of multiple bridge structural configurations, generated through summary statistics of geometrical and material properties, which can be typically drawn from national bridge inventories. The obtained bridge portfolio can then be characterized in terms of eigenvalue analyses output, which will be useful to either identify the fundamental period of vibration or define pushover curve loads for nonlinear seismic demand estimation methods. In fact, in the past, a large number of simplified equations to estimate the fundamental period of vibration have been proposed and are commonly used for buildings (e.g. [21, 22]) whereas the absence of similar equations for bridges is well known. This paper intends to contribute to fill such a gap by proposing simplified equations as function of one or more parameters strongly correlated to the fundamental period. The fundamental period of vibration becomes also handy to e.g. carry out proper selection of records according to recent methodologies, such as the conditional spectrum method [23], also successfully applied to bridges [24]. Such a selection guarantees reliable performance points for any employed nonlinear static procedure or seismic input for nonlinear dynamic analyses.

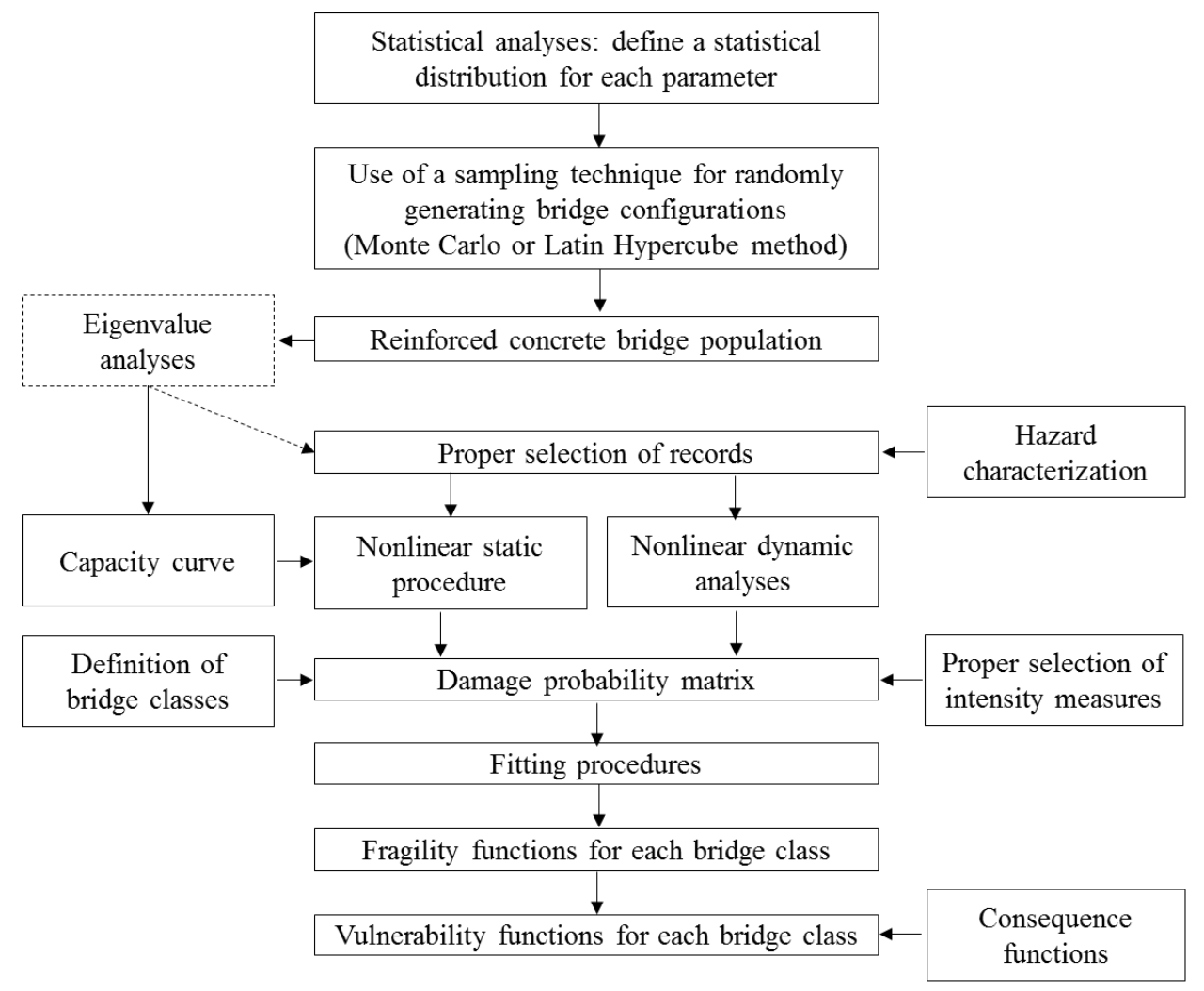

Figure 1. General framework

Once bridge classes are identified, a proper intensity measure and damage limit states are selected so that the damage probability matrix can be derived. This matrix becomes the input of a fitting procedure developed to identify the main fragility function parameters. Therefore, 
the framework is very much dependent on randomly simulated bridges, featuring different geometrical configurations. Indeed, the randomness that characterizes the simulated population is exclusively related to the structural layout of the pier heights and deck spans, together with the variability associated to the material properties. With this portfolio it is possible to establish, through regression analysis, empirical equations that relate geometrical properties with corresponding structural response and, afterwards, fragility. Such equations may then find useful application in expedite risk assessment methodologies or national design codes.

\section{STATISTICS AROUND BRIDGE PROPERTIES}

In order to provide the input for numerical simulation of bridge populations, the available information of the bridge stock under investigation has been statistically characterized. Statistical distributions were assigned to each parameter deemed relevant to the behaviour of reinforced concrete bridges. For the selected geometrical and material properties, the main statistical quantities were evaluated from the sample. Chi-Square and/or KolmogorovSmirnov goodness-of-fit (GoF) tests were applied to assign normal, lognormal, gamma, exponential or Weibull distributions, according to typical alpha values $(0.05,0.10$ and 0.20$)$. At an initial stage, all the properties available from the source database were statistically characterized through GoF testing. Afterwards, bridge configuration sampling and empirical relationships were carried out only for the properties that were relevant for a common RC bridge configuration, as detailed in Section 4, namely piers height, total length of bridges, span length, section diameter, reinforcing bars' Young modulus, concrete compressive strength.

\subsection{Chi-Square Goodness-of-Fit test}

This test should be employed under specific conditions: it requires a sufficient sample size, only binned data are allowed and a minimum expected frequency is required. Given that the selection of the number of bins affects the value of the test, some rules (e.g. Sturge's rule, Normal reference rule and Scott's rule) suggest the most appropriate bin widths in order to minimize the squared error in the approximation of the Chi-Square test. The minimum expected frequency is unanimously established by literature equal to five, and if this minimum requirement is not reached, the combination of adjacent bins in the tails is applied. The subsequent overlapping of the theoretical probability density function of the assumed distribution on each histogram shows if there is probabilistic agreement; if there is not, the null hypothesis, which asserts that the data follows the pre-specified distribution, is most likely incorrect.

\subsection{Kolmogorov-Smirnov Goodness-of-Fit test}

The Kolmogorov-Smirnov GoF test belongs to the class of distance tests and is among the best distance tests for small samples, yet it is still reliable for large ones. The test is developed starting from the assumption of an empirical distribution, characterized by parameters estimated from the data set or from expert judgment. The comparison between theoretical and empirical CDFs follows. If the two CDF curves agree from the probabilistic point of view, the assumed distribution is accepted. Such comparison consists of defining the maximum vertical distance between the two curves and the p-value based on the maximum distance. The Kolmogorov-Smirnov test is widely used in practice and it works properly even if the sample size is small; hence it becomes particularly useful for the statistical characterization of geometrical and material properties of existing structures, such as the ones herein investigated. 


\subsection{Goodness-of-Fit testing with data truncation}

A non negligible percentage of the data for the different variables was located at the tails of the corresponding distribution functions. The identification of outliers was therefore necessary. One way to recognize outliers is by using box plots, which synthetically represent all the data and group it in quartiles. The variability outside the upper and lower quartiles (respectively q3 and $\mathrm{q} 1$ in Figure 2) was assumed as 1.5 times the interquartile range (IQR) and is represented by whiskers. The data outside the whiskers length is identified as outliers. The range of data that excludes outliers is the so-called inner fence and it was used as input for the statistical tests [25].

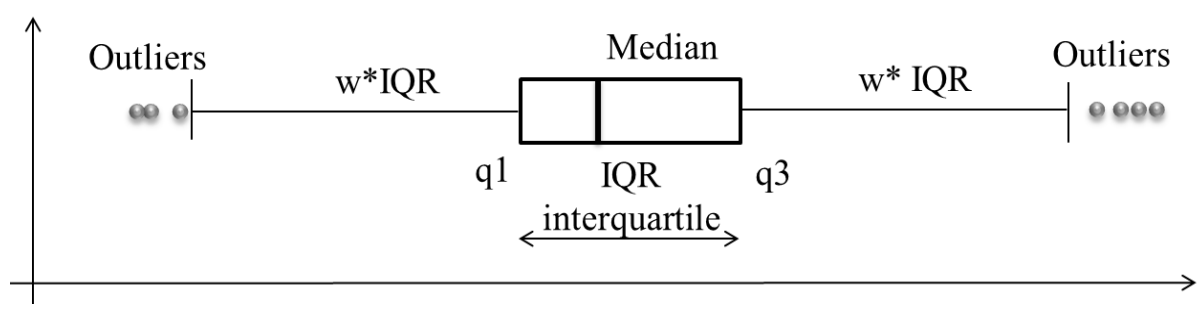

Figure 2. Typical box plot for identifying outliers

In the present work, the variables that did not satisfy goodness of fit tests were analysed through box plots and in some cases, to better fit the theoretical statistical distribution, a whisker length of 3.0 was allowed. All the distribution parameters were accordingly derived.

\section{DEFINITION OF A TYPICAL BRIDGE STOCK}

The statistical treatment of geometrical and material properties was conducted by selecting, among the available data, the most relevant parameters from the structural seismic response viewpoint. The main bridge typology considered corresponds to Italian reinforced concrete bridges built between the 1970s and 1990s, when the major development of the highway network took place. In most cases, such bridges are characterized by simply supported deck or monolithic connections (Figure 3). About 30\% of the structures exhibit circular or rectangular pier cross sections, whereas $35 \%$ include several section shapes such as octagonal or composite sections. The pier-to-deck connection is typically of the concrete-concrete or elastomeric bearings or other devices type.

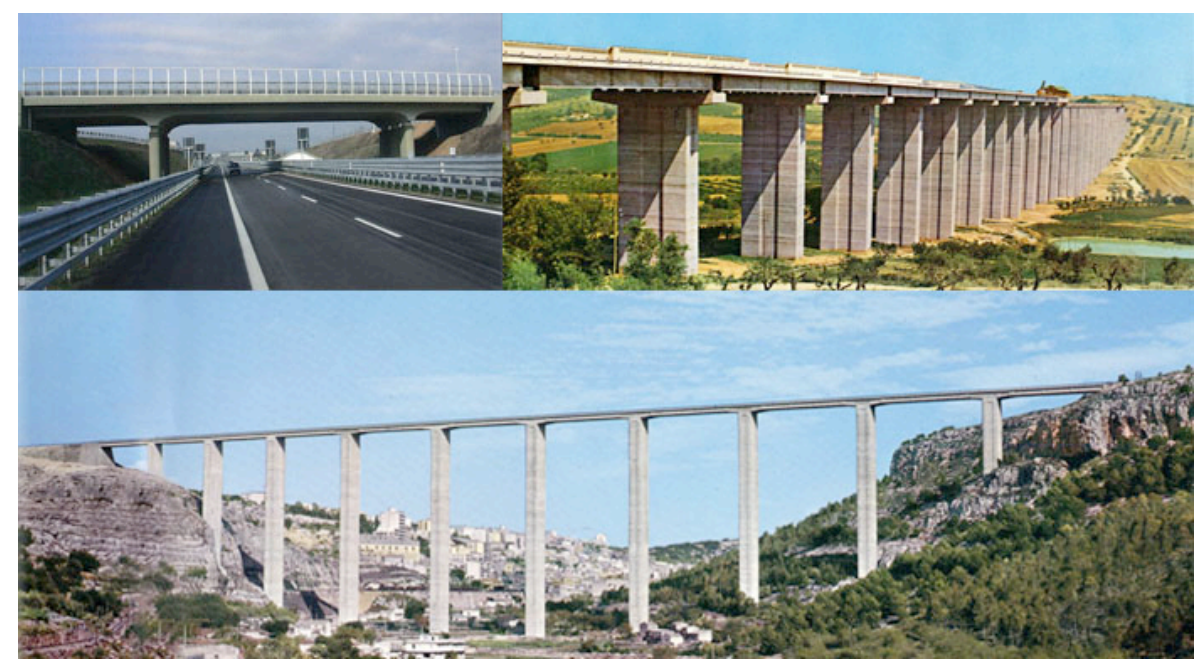

Figure 3. Typical Italian highway bridge. [26] 
A case study made up of nearly five hundred Italian bridges (the exact number is 458), illustrated in Figure 4, was built up with a view to the simplified seismic vulnerability estimates for RC bridges by using parameterized structural behaviour relationships and future fragility curves.

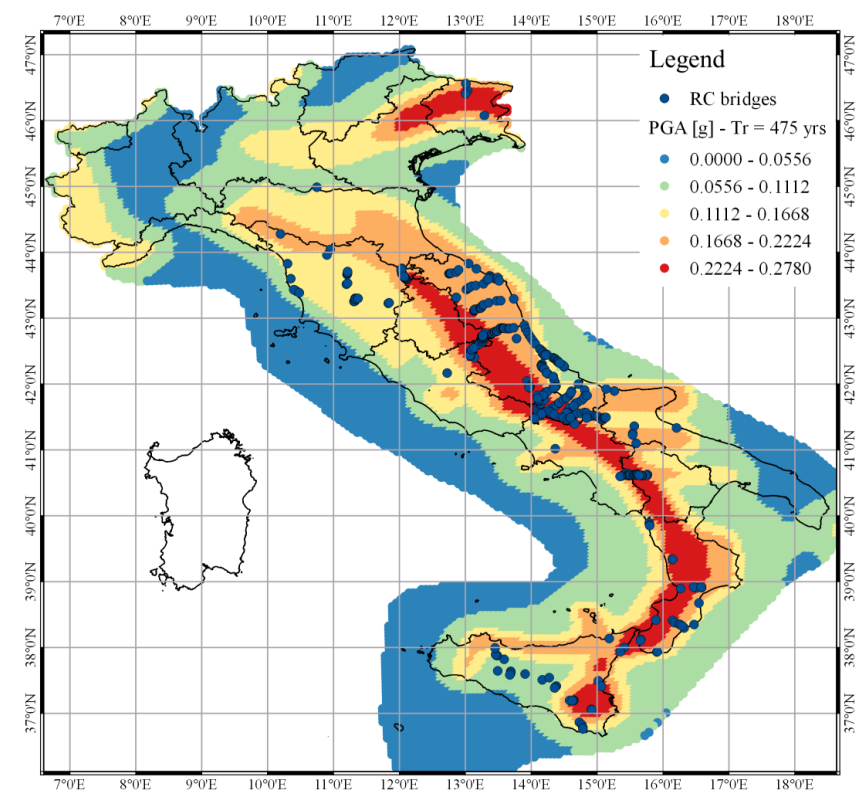

Figure 4. Case study: Italian reinforced concrete bridge population

Ideally, within a seismic risk assessment context, the definition of fragility curves of each bridge should be carried out. However, to avoid the considerable time demand of such an approach, the definition of bridge classes for a certain country would be reasonable if one assumes that the characteristics of bridges in a certain region are typically similar. Furthermore, the approach followed in the present section is based on general geometric and material information provided by a database, which is not enough to define an exhaustive characterization of different bridge classes.

At this point, the portfolio characterization in terms of random simulation of bridge properties can follow two macro strategies. The first is to consider specific geometric configurations with a specific layout (predefined number of piers, spans and regularity level) and then establish a distribution for every variable of the layout to perform simulations. The second, that is adopted here, considers the number of piers, spans and regularity level (pier heights) as random variables, defined statistically.

As such, the Italian bridges represented in Figure 4 were gathered in a single macro bridge category, corresponding to straight multi-span continuous reinforced concrete bridges. These bridges were modelled using the same deck and pier cross section type and featured no isolation at the pier-to-deck connection level. Abutments were considered as of very high stiffness hence nearly fully fixed. Furthermore, curved bridges were assumed as straight and were grouped in that same class, whilst arch bridges were considered as part of a different configuration type hence not included in the present study. The available geometrical parameters for each bridge, schematically depicted in Figure 5, were individually analysed and general inference on bridge class was made when the full set of bridge properties was not available.

Construction type, column height, span length, number of spans, superstructure area, radius of curvature in plan, main geometrical dimension of the column section, steel bars tensile strength, steel bars Young Modulus were the available parameters that provide insight to- 
wards the typical geometric configuration and material properties of the selected bridges. No specific information related to the seismic design was available. A possible indicator to determine whether the bridges were seismically designed or not could be the year of construction. With respect to the bridges for which the construction year is known, $65 \%$ were built before 1970; 25\% between 1970 and 1980, and 10\% after 1980 . Therefore, all the bridges were conservatively assumed as non seismically designed.

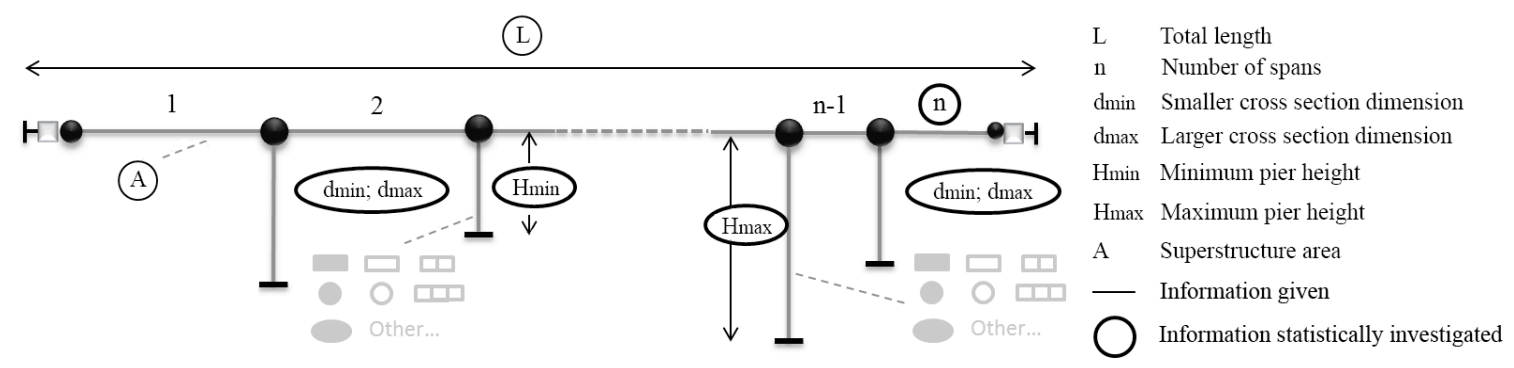

Figure 5. Available information on the case study bridges

For most of the properties, statistical details were investigated using the conventional statistical tools for distribution testing, described in Section 3, to define input for numerical simulation. Specific attention was paid to discrete data, for which different statistical treatment was required, particularly for the number of spans variable, which only admits integer values. In fact, even though the use of Poisson regression would be required, the resulting inappropriate short spans obtained with such an approach led the authors to consider instead the relationship between number of spans and total length, as given in Table 1.

\begin{tabular}{|l|l|l|l|}
\hline Variable & Distribution & Distr. parameters & Statistical tests \\
\hline Pier height $[\mathrm{m}]$ & Lognormal & $\begin{array}{l}\text { Mean }(\log ): 1.95 \\
\text { std: } 0.8275\end{array}$ & $\begin{array}{l}\text { Chi-sq. not sat. } \\
\text { K-S: 10\%, sat. }\end{array}$ \\
\hline Total length $[\mathrm{m}]$ & Lognormal & $\begin{array}{l}\text { Mean }(\log ): 5.10 \\
\text { std: } 1.145\end{array}$ & $\begin{array}{l}\text { Chi-sq. not sat. } \\
\text { K-S not sat }\end{array}$ \\
\hline Span length $[\mathrm{m}]$ & Normal & $\begin{array}{l}\text { Mean: } 31.18 \\
\text { std: } 11.527\end{array}$ & $\begin{array}{l}\text { Chi-sq. not sat. } \\
\text { K-S not sat. }\end{array}$ \\
\hline Average span length $[\mathrm{m}]$ & Normal & $\begin{array}{l}\text { Mean: } 29.936 \\
\text { std: } 12.174\end{array}$ & $\begin{array}{l}\text { Chi-sq. not sat. } \\
\text { K-S not sat. }\end{array}$ \\
\hline Number of spans & Regression & $\begin{array}{l}\text { \# spans }=1.483+0.0273 * \text { Total length } \\
\mathrm{R}^{2}=0.89\end{array}$ \\
\hline Superstructure area $\left[\mathrm{m}^{2}\right]$ & Lognormal & $\begin{array}{l}\text { Mean }(\log ): 7.88 \\
\text { std: } 0.9087\end{array}$ & $\begin{array}{l}\text { Chi-sq.: 5\%, sat. } \\
\text { K-S: 10\%, sat. }\end{array}$ \\
\hline
\end{tabular}

Table 1. Statistical distributions - geometrical layout properties

The superstructure width, required for structural modelling and not included in the set of available variables, was derived from the superstructure area, considering a trapezoidal widespread Italian deck cross section with the assumption of a solid cross section. The depth of the section was defined in line with real bridges, taking into account the average span length. In particular, for an average span length of $20 \mathrm{~m}$, a reasonable value of deck depth was registered to be $1.30 \mathrm{~m}$; whilst in the case of $40 \mathrm{~m}$, the deck depth assumed was $2.20 \mathrm{~m}$. In order to randomly generate superstructure widths, a linear trend was assumed and inference of data was then applied. The 458 observations for superstructure width were then derived from the corresponding superstructure area and total bridge length, whose fitting to the most common statis- 
tical distributions were tested through Chi-squared and Kolmogorov-Smirnov GoF tests. Although the test was not satisfied at either $99 \%$, at $90 \%$ or at $95 \%$ confidence levels, the best fit was observed to be obtained with a lognormal distribution, without truncation of data. The random data was generated between $4.7 \mathrm{~m}$ and $34.7 \mathrm{~m}$, with a mean value of $12.5 \mathrm{~m}$ and coefficient of variation of $29.1 \%$.

The pier cross section characterization was carried out in terms of solid sections, particularly circular cross sections, which were used for the random simulation in Section 5. As for material properties, elevation compressive strength, bar tensile strength and reinforcing bars Young Modulus statistical distributions were available.

Finally, it is worth noting that when carrying out data truncation of the geometrical properties that exhibited a number of outliers, the distribution parameters sometimes significantly changed.

\section{RANDOM SAMPLING: GENERATION OF BRIDGE POPULATIONS}

Following the statistical characterization of the geometrical and material properties, the random generation of bridge populations can be carried out by simulating possible values from the probability distribution functions, adjusted to the different variables described in Section 4. To accomplish this, two random simulation schemes, sharing the same underlying concepts, were selected: standard and Latin Hypercube-based Monte Carlo. A sufficient number of iteration steps was ensured to guarantee that sampled parameters approximate the initially assumed probability distribution functions. Accurate results for output distributions depend on how complete the sampling from the input distributions is, thus more efficient methods are those that reach this objective with the lower number of iterations. The most popular sampling algorithm in engineering applications is standard Monte Carlo (MC), which generally requires a large number of iteration steps, whereas a more recent sampling strategy proposed is the Latin Hypercube Sampling-based Monte Carlo (LHS) that converges faster on the statistics of the input distribution. Needless to say, the selection of a proper sampling method is a crucial phase of nonlinear engineering data analysis applications, as it affects both computational onus and accurateness of results.

\subsection{Sampling with standard Monte Carlo scheme}

Monte Carlo is a widely spread technique applied to many problems that require the extraction of random or pseudo-random numbers to sample from a probability distribution. In its crude or standard version, the Monte Carlo sampling technique assumes that any given sample may fall anywhere within the range of the input distribution. Its main drawback is that when probability distributions are noticeably skewed, it can present outcomes of low probability, together with clustering. These limitations have incited researchers to develop new sampling schemes, such as the Latin Hypercube-based Monte Carlo, discussed ahead. In the study presented herein, Monte Carlo sampling was tested for the generation of a population set of 1000 bridges, assuming as variables the pier height (lognormal distribution), span length (normal distribution), total bridge length (lognormal distribution), section diameter (normal distribution), longitudinal reinforcement yield strength (normal distribution), transverse reinforcement yield strength (normal distribution) and steel Young Modulus (normal distribution).

\subsection{Sampling with Latin Hypercube scheme}

The Latin Hypercube sampling scheme is a particular case of the Monte Carlo method and consists of the stratification of the input cumulative distribution function (CDF). This CDF is 
subdivided into equal intervals from which the samples are randomly drawn without replacement, retracing the input probability distribution [27]. The input parameters of each simulation constitute the rows of an NxK matrix ( $\mathrm{N}$ is the number of the numerical simulations and $\mathrm{K}$ is the number of parameters), obtained according to the inverse target distribution of the parameter associated with the column involved [28].

Even though the LHS-based method exhibits the advantage of reducing computational time demand, it does not allow the specification of parameter lower and upper bounds nor generating a new single bridge configuration. To be consistent with the bounds specified in the standard Monte Carlo method, for each parameter, such a drawback was overcome by imposing a greater number of simulations and by eliminating the entire input rows associated to those out of range values. Moreover, in order to avoid unrealistic negative parameter values, the possible entire input rows of the aforementioned $\mathrm{N}$ by $\mathrm{K}$ matrix with negative values were removed. Very few simulations did not respect the imposed restrictions therefore the described assumptions were considered acceptable.

According to the aforementioned rationale, assumptions, steps, and available information (Figure 5), a sample of 100 bridge configurations was generated. This sampling size is in agreement with recent studies in the field [29], which focused on the employment of LH sampling to the probabilistic seismic assessment of RC bridges. The distributions of the fundamental period in the transverse direction of the bridge populations generated with standard and LH-based Monte Carlo sampling are shown in Figure 6.
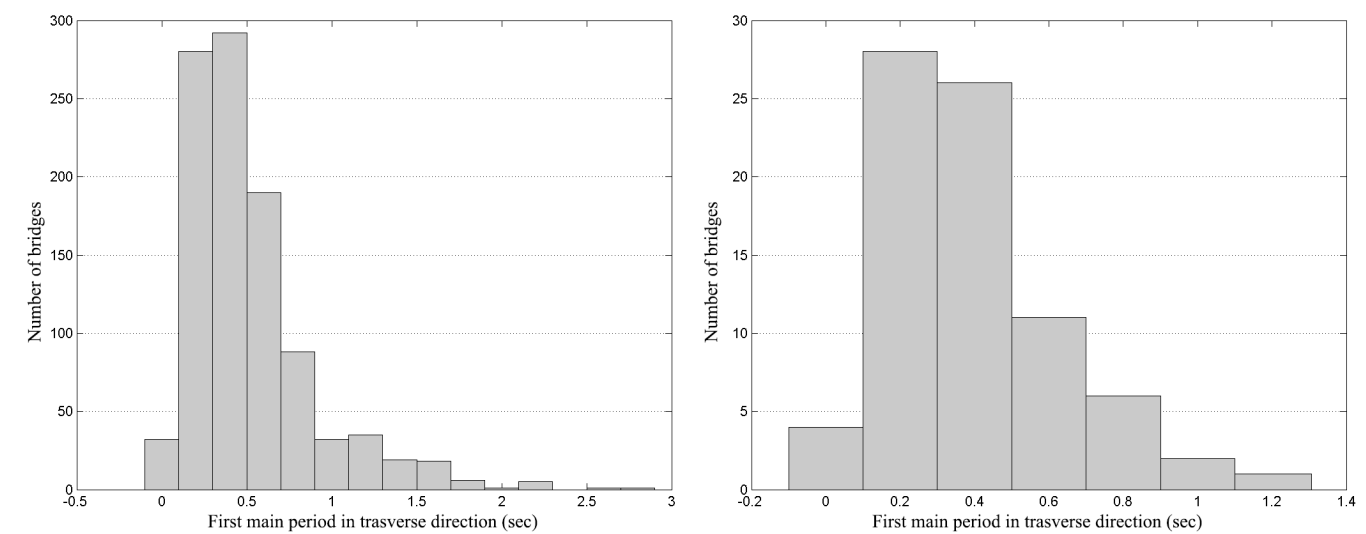

Figure 6. Fundamental transverse period of bridges randomly generated with (left) standard and (right) Latin Hypercube-based Monte Carlo sampling schemes.

\section{PERIOD ESTIMATION - REGRESSION ANALYSIS}

In seismic analysis, the knowledge related to the fundamental period of vibration is very important as it represents a parameter capable of providing relevant information related to the global demand on a structure under a given ground motion. Structural mass, stiffness and strength are the variables that primarily affect such a dynamic behaviour parameter. However, their knowledge is not always immediate, which renders many times useful a relationship between the period and easily obtainable properties, such as geometrical information. This relationship will in turn be useful to get an accurate estimate whenever detailed data for a certain bridge is missing. The lack of this sort of studies for bridges, as opposed to buildings, led to the identification of one or more parameters strongly correlated to the fundamental period of vibration, through an analytical procedure based on the numerical simulation of bridge portfolios. Single variable and multivariate regression analyses were carried out on the randomly generated bridge populations for the development of the period estimation relationship. 


\subsection{Existing bridge period relationships}

Eurocode 8 proposes different formulae for the structural period of vibration based on stiffness and displacements, differentiated for the cases of rigid and flexible decks. A recent methodology [30] produced simplified capacity curves to be used for seismic loss assessment of bridges. A bridge population of twenty-one assets was considered as case study. It was characterized by two deck lengths $(200 \mathrm{~m}$ and $400 \mathrm{~m})$, three different types of abutments and different geometrical layouts, corresponding to different regularity classes: regular, semiregular, and irregular. The initial period in the transverse direction, corresponding to the equivalent single-degree-of-freedom system's yielding point, resulted as a relevant parameter for characterizing simplified capacity curves, thus a preliminary trend line was drawn for defining its relationship with deck length. The number of bridges used was very limited hence the need for an improved relationship of that sort is evident. When detailed information of existing bridges is not readily available, the properties that can be easily obtained are of geometric nature. For such reason, at this initial stage, only their correlation with the fundamental period is considered.

\subsection{Bridge modelling}

Each case-study bridge was modelled in [31], which enabled the creation of an automatized procedure controlled by an external script [32], capable of generating and updating model files for each run. The deck was modelled as a continuous elastic element, located right at the pier top, with lumped masses and with geometrical properties referred to its centre of mass, connected through rigid link elements. Considered the bridge stock characteristics, circular solid sections were assigned to each pier. With respect to material modelling, KentScott-Park model with zero tensile strength was assigned to represent concrete behaviour, accounting for confinement factor in case of concrete cores. The confinement level was evaluated for each bridge component through equations proposed by Mander, since neither KentPark's model nor its modified version provided information about the confining effects of spiral hoops. A bilinear model was assumed for steel, with no isotropic hardening. On the other hand, kinematic hardening was considered through the ratio between post-yield tangent and initial elastic tangent, i.e. strain-hardening ratio equal to 0.002 . The connection between the piers and the deck was assured by shear keys that were modelled with rigid zero length elements and equal-degree-of-freedom connections. As far as abutments were concerned, a simplified model made up of very high stiffness springs with bilinear response along horizontal directions and fully restrained at the ground was employed.

\subsection{Parametric study}

Trying to fill the identified gap in the current state-of-the-art, a relationship between the fundamental period of the defined bridge class and relevant geometrical parameters was sought using eigenvalue analysis. Such approach made use of the randomly generated bridges, guaranteeing the consideration of a large breadth of different structural configurations. The representation of the fundamental period versus various geometrical parameters (tallest pier height, shortest pier height, average pier height, bridge length, average pier height) for 1000 bridges generated with standard Monte Carlo simulation was carried out in accordance with the statistical distributions proposed in Section 4. This did not evidence a clear nor satisfactory trend between fundamental period and each parameter considered individually. This is certainly due to the fact that several variables are being randomly considered and, as such, multivariate regression is envisaged with a view to an improvement in the strength of such a relationship. Nevertheless, pier height and average pier height parameters seemed to yield a 
stronger correlation with the fundamental period, which preliminary indicates a possible influence by factors as regularity and bridge length.

With respect to the set of 100 bridges generated with LH-based Monte Carlo sampling, a screening of the different configurations was carried out to eliminate those including characteristics without physical meaning (e.g. negative pier heights). After such filtering, the remaining number of configurations was 78 .

Considering multivariate regression, the analyses were carried out using the standard (1000) and LH-based Monte Carlo (78) sampling generated bridges. The corresponding equations and plots are presented, respectively, in Tables 2 and 3. The results denote a trend very similar to the standard Monte Carlo based results. Indeed, the comparison between the results obtained from multivariate regression analyses applied to the bridge populations associated with the two sampling procedures are summarized in Table 4. Such results demonstrate that the LH-based sampling scheme provides very close results, even slightly better, to those obtained with the standard Monte Carlo algorithm.

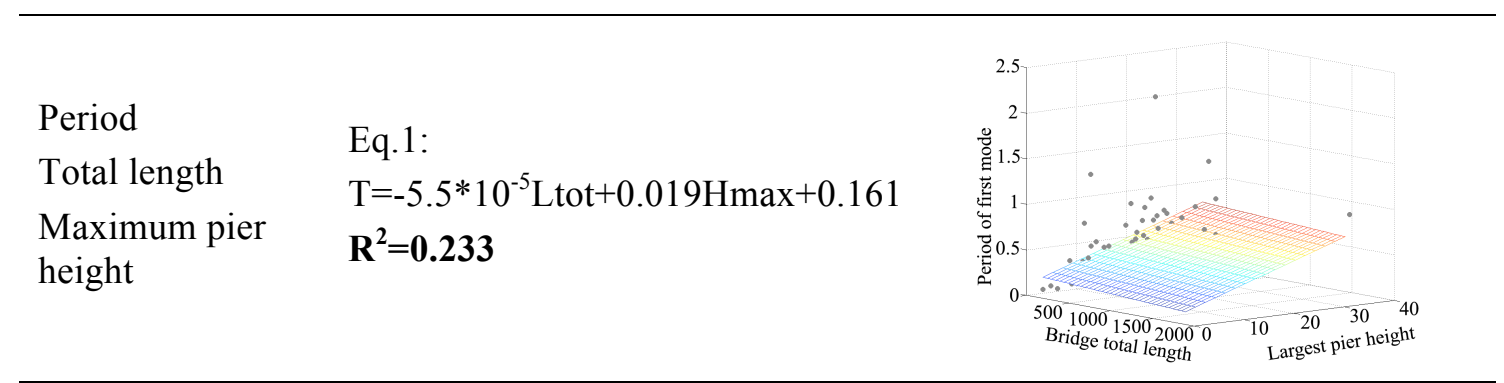

\section{Period}

Maximum pier height

Section diameter

Eq.1:

$\mathrm{T}=0.017 \mathrm{Hmax}-0.225 \mathrm{Dsec}+0.718$

$\mathbf{R}^{2}=\mathbf{0 . 5 9 4}$

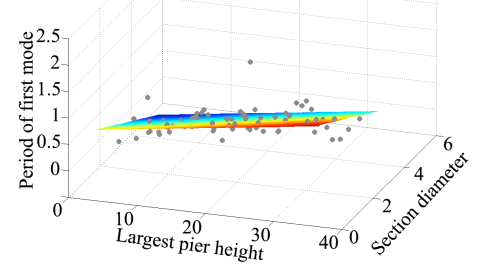

\section{Period}

Average pier

Eq.1:

height

Section diameter

$\mathrm{T}=0.054 \mathrm{Hav}-0.215 \mathrm{Dsec}+0.523$

$\mathbf{R}^{2}=\mathbf{0 . 6 5 6}$

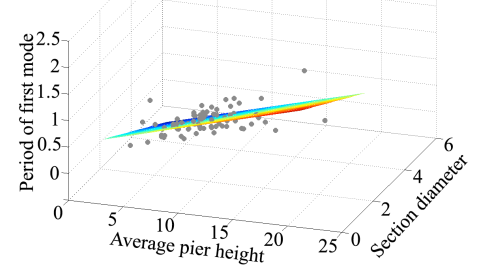

Table 2. Multivariate regression for 1000 bridges using standard Monte Carlo simulation

The final set of regression analysis tests considered the ratio between the two best performing variables (average pier height and section diameter), $\mathrm{H}_{\mathrm{av}} / \mathrm{D}_{\mathrm{sec}}$, which better fitted the results obtained with numerical simulation. Further improvement of the quality of the fitting was obtained by:

- carrying out robust regression, which assigns a different weight to each point while performing the regression;

- considering the semi-studentized residuals in order to identify the presence of outliers. According to a rough rule of thumb, suggested in [25], semi-studentized residuals with 
absolute value of four or more are to be considered outliers hence they were not included when deriving regression equations.

Period

Total length

Maximum pier

height
Eq.1:

$\mathrm{T}=-5.5^{*} 10^{-5} \mathrm{Ltot}+0.019 \mathrm{Hmax}+0.161$

$\mathbf{R}^{2}=\mathbf{0 . 2 3 3}$

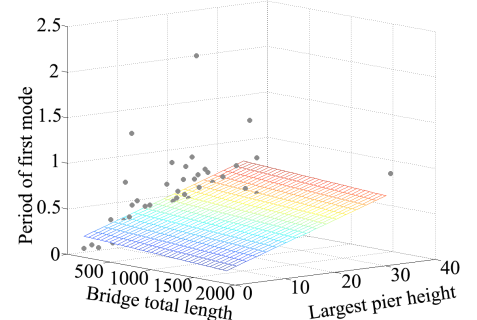

\section{Period}

Maximum pier height

Section diameter
Eq.1:

$\mathrm{T}=0.017$ Hmax $-0.225 \mathrm{Dsec}+0.718$

$\mathbf{R}^{2}=\mathbf{0 . 5 9 4}$
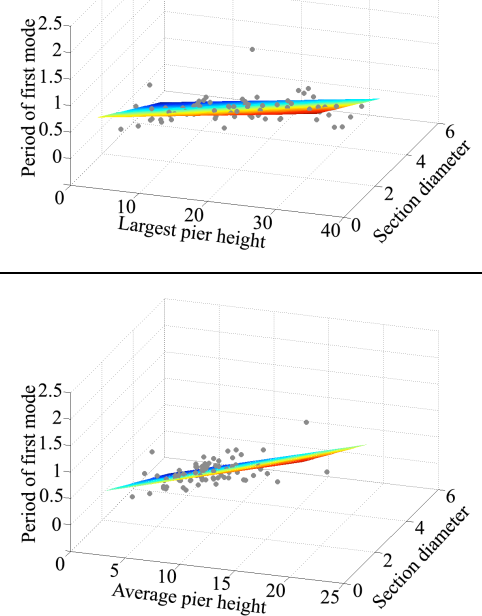

Table 3. Multivariate regression for 78 bridges using LH-based Monte Carlo sampling

\begin{tabular}{|c|c|c|}
\hline Relationship & $\begin{array}{l}\text { Standard Monte Carlo (1000 } \\
\text { simulations) }\end{array}$ & $\begin{array}{l}\text { LHS-based Monte Carlo ( } 78 \\
\text { simulations) }\end{array}$ \\
\hline Period & Eq.1: & Eq.1: \\
\hline Total length & $\mathrm{T}=2.0^{*} 10^{-4} \mathrm{Ltot}$ & $\mathrm{T}=-5.5^{*} 10^{-}$ \\
\hline $\begin{array}{l}\text { Maximum piers } \\
\text { height }\end{array}$ & $\begin{array}{l}+0.015 \mathrm{Hmax}+0.239 \\
\mathbf{R}^{\mathbf{2}}=\mathbf{0 . 1 6 3}\end{array}$ & $\begin{array}{l}{ }^{5} \mathrm{Ltot}+0.019 \mathrm{Hmax}+0.161 \\
\mathbf{R}^{\mathbf{2}}=\mathbf{0 . 2 3 3}\end{array}$ \\
\hline $\begin{array}{l}\text { Period } \\
\text { Maximum piers } \\
\text { height } \\
\text { Section diameter }\end{array}$ & $\begin{array}{l}\text { Eq.1: } \\
\mathrm{T}=0.016 \text { Hmax-0.229Dsec }+0.977 \\
\mathbf{R}^{\mathbf{2}}=\mathbf{0 . 5 9 1}\end{array}$ & $\begin{array}{l}\text { Eq.1: } \\
\mathrm{T}=0.017 \text { Hmax-0.225Dsec }+0.718 \\
\mathbf{R}^{\mathbf{2}}=\mathbf{0 . 5 9 4}\end{array}$ \\
\hline $\begin{array}{l}\text { Period } \\
\text { Average piers } \\
\text { height } \\
\text { Section diameter }\end{array}$ & $\begin{array}{l}\text { Eq.1: } \\
\mathrm{T}=0.048 \text { Hav- } 0.303 \mathrm{Dsec}+0.777 \\
\mathbf{R}^{2}=\mathbf{0 . 5 9 0}\end{array}$ & $\begin{array}{l}\text { Eq.1: } \\
\mathrm{T}=0.054 \mathrm{Hav}-0.215 \mathrm{Dsec}+0.523 \\
\mathbf{R}^{\mathbf{2}}=\mathbf{0 . 6 5 6}\end{array}$ \\
\hline
\end{tabular}

Table 4. Comparison between multivariate regression results for the two sampling schemes 
The plots and equations representing the improved relationship, associated with both Latin Hypercube and Monte Carlo sampling schemes, are illustrated in Table 5. When considering the ratio between average pier height and section diameter, the improvement in the period of vibration parametric characterization is notorious, whether standard or LH-based Monte Carlo sampling is employed. The quality and strength of the identified correlation is now at a level able to provide enough confidence in the use of the corresponding formulae. Indeed, a very quick estimate of the period of vibration of bridges with the characteristics used in this study can be taken as one tenth of the ratio between the average pier height and the pier cross section diameter.

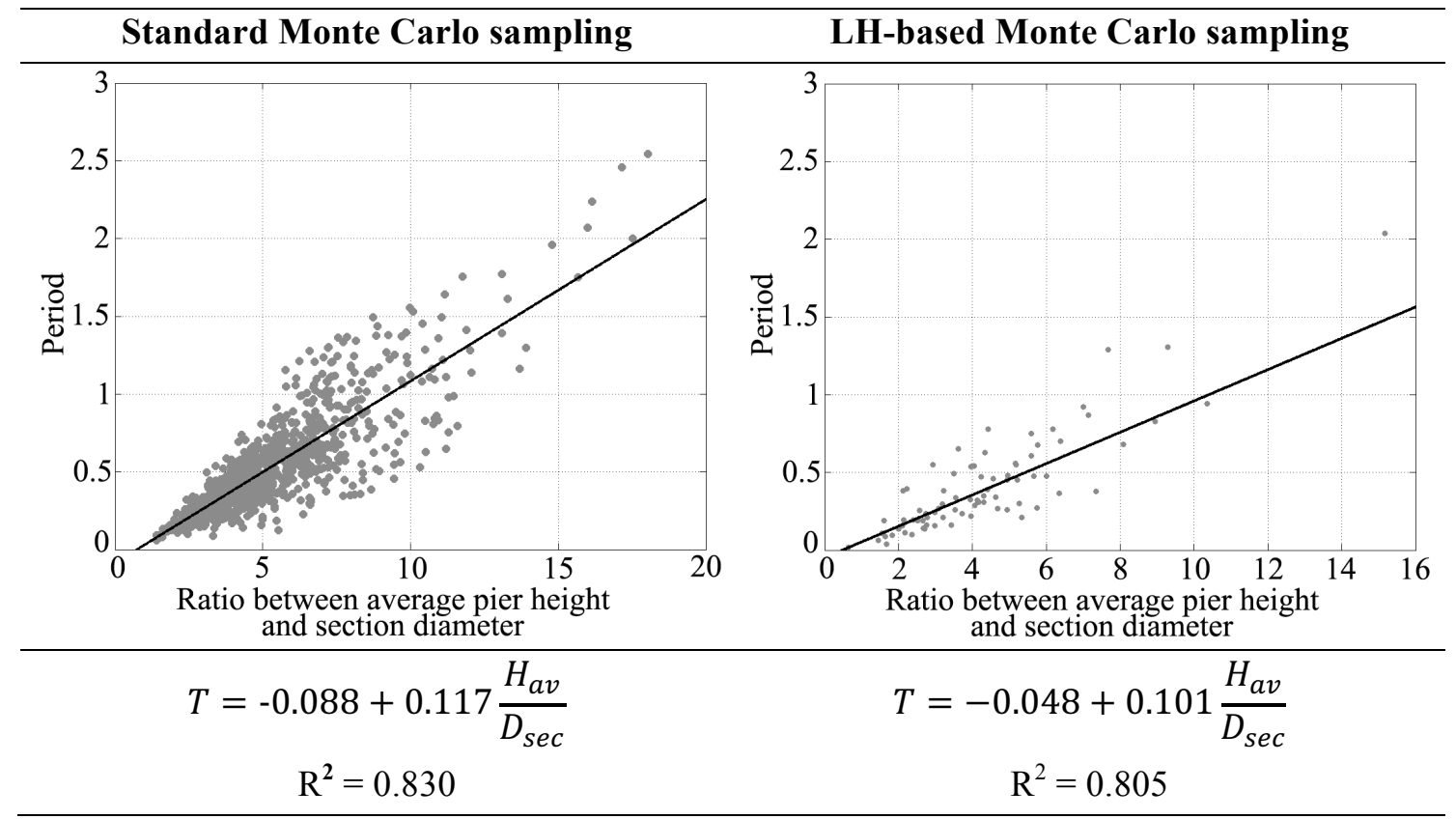

Table 5. Fundamental period versus ratio (average pier height / section diameter)

\section{CONCLUSIONS}

The study presented in this paper shows, as main contribution, a relationship to estimate the period of vibration of a typical class of Italian reinforced concrete bridges, based on easily measurable geometrical parameters. with a view to seismic assessment studies. Such bridge typology features simply supported continuous deck, nearly fixed abutments and piers with circular cross section. The geometrical variability was defined in terms of pier and span layout (number and, respectively, height or length) and material properties. The parametric characterization is useful to e.g. rapid loss assessment studies of bridge portfolios. In such a context, expedite estimates of structural characteristics are useful, especially when limited information is available. In specific, the period of vibration is intended to provide simplified seismic structural demand information, enabling also future development of fragility curves by classes.

The geometrical and material properties characterization related to existing bridges was successfully carried out through a thorough assignment of statistical distributions based on goodness-of-fit tests and outlier elimination techniques. Using such distributions, the random generation of a large number of bridges was then performed by applying two different sampling schemes: standard Monte Carlo, characterized by fully randomness, and Latin Hypercube-based Monte Carlo, based on stratified randomness. Both procedures proved to be effective in sampling bridge populations to obtain the desired parametric relationships through robust nonlinear regression analysis. For both sets of generated bridges, corresponding to the 
different simulation techniques, the determination coefficients were similar and higher than 0.8 , a fair result from an engineering viewpoint. Taking into account the reduced computational onus without loss of accuracy, the Latin Hypercube-based Monte Carlo sampling scheme revealed itself more advantageous and is therefore proposed as the preferable solution for numerical simulation in engineering applications of this sort.

For what concerns the specific relationship between the period of vibration and the geometrical parameters of the bridges, a simplified equation has been successfully proposed. From the authors perspective, it can be employed as a starting step for the expedite assessment of existing bridges of predefined bridge classes (with corresponding e.g. fragility curves). For the considered class, the period of vibration is seen as typically related to the ratio between the average pier height and the pier cross section diameter, properties that can be easily obtained through visual inspection or even remote techniques (e.g. GIS tools). It is believed that the work herein presented overcomes its inherent limitations when considering that current risk assessment studies, carried out in academic and industry, are based on fragility and exposure models built upon proxies with less technical content (e.g. census data). Future developments of the present work will indeed investigate the possibility to extend the proposed period equation to other branches of bridge taxonomy i.e. structural bridge classes. Important developments will also concern the consideration of code-prescribed levels of seismic design in terms of strength and ductility in the classification of bridges.

\section{REFERENCES}

[1] M.T. Bensi, A Bayesian Network Methodology for Infrastructure Seismic Risk Assessment and Decision Support, $\mathrm{PhD}$ thesis, 2010.

[2] A. Sextos, ICT applications for new generation seismic design, construction and assessment of bridges. Structural Engineering International: Journal of the International Association for Bridge and Structural Engineering (IABSE), 24(2), 173-183, 2014.

[3] C. Casarotti, R. Monteiro and R. Pinho, Verification of spectral reduction factors for seismic assessment of bridges. Bulletin of the New Zealand Society for Earthquake Engineering, 42(2), 111, 2009.

[4] R. Pinho, R. Monteiro, C. Casarotti, R. Delgado, Assessment of continuous span bridges through nonlinear static procedures. Earthquake Spectra, 25(1), 143-159, 2009.

[5] M. Kohrangi, R. Bento, M. Lopes, Seismic performance of irregular bridgescomparison of different nonlinear static procedures. Structure and Infrastructure Engineering, 1-19, 2014.

[6] R. Monteiro, R. Delgado, R. Pinho, Probabilistic seismic assessment of RC bridges: Part I - Uncertainty models. Structures, 5, 258-273, 2016.

[7] R. Monteiro, R. Delgado, R. Pinho, Probabilistic seismic assessment of RC bridges: Part II - Nonlinear demand prediction. Structures, 5, 274-283, 2016.

[8] M. Fragiadakis, D. Vamvatsikos, M. Aschheim, Application of Nonlinear Static Procedures for the Seismic Assessment of Regular RC Moment Frame Buildings. Earthquake Spectra, 30(2), 767-794, 2014. 
[9] R. Monteiro, M. Marques, G. Adhikari, C. Casarotti, R. Pinho, Spectral reduction factors evaluation for seismic assessment of frame buildings. Engineering Structures, 77, 129-142, 2014.

[10] R. Pinho, M. Marques, R. Monteiro, C. Casarotti, R. Delgado, Evaluation of nonlinear static procedures in the assessment of building frames. Earthquake Spectra, 29(4), 1459-1476, 2013.

[11] R. Monteiro, P. Ceresa, V. Cerchiello, J. Dabeek, A. Di Meo, B. Borzi, Towards integrated seismic risk assessment in Palestine - Application of the city of Nablus. M. Papadrakakis, V. Papadopoulos, G. Stefanou, V. Plevris eds. VII European Congress on Computational Methods in Applied Sciences and Engineering (ECCOMAS Congress), Crete Island, Greece, 5-10 June, 2016.

[12] I. Grigoratos, J. Dabeek, M. Faravelli, A. Di Meo, V. Cerchiello, B. Borzi, R. Monteiro, P. Ceresa, Development of a fragility and exposure model for Palestine - Application to the city of Nablus. Proceedings of the The World Multidisciplinary Civil EngineeringArchitecture-Urban Planning Symposium, Prague, 13-17 June, 2016.

[13] K. Mackie, B. Stojadinovic, Probabilistic Seismic Demand Model for California Bridges. Journal of Bridge Engineering, 6(6), 468-480, 2001.

[14] E. Choi, R. DesRoches, B. Nielson, Seismic Fragility of Typical Bridges in Moderate Seismic Zones. Engineering Structures, 26(2), 187-199, 2004.

[15] A.C. Cornell, F. Jalayer, R.O. Hamburger, Probabilistic Basis for 2000 SAC Federal Emergency Management Agency Steel Moment Frame Guidelines. Journal of Structural Engineering, 128(4), 526-532, 2002.

[16] B. Nielson, R. DesRoches, Analytical seismic fragility curves for typical bridges in the central and southeastern United States. Earthquake Spectra, 23(3), 615-633, 2007.

[17] C. Zelaschi, D. Forcellini, G. De Angelis, R. Monteiro, Performance based earthquake engineering approach applied to bridges in a road network. Proceedings of the 5th ECCOMAS Thematic Conference on Computational Methods in Structural Dynamics and Earthquake Engineering (COMPDYN), Crete, Greece, May 25-27, 2015.

[18] M. Fragiadakis, D. Vamvatsikos, M. Karlaftis, N. Lagaros, M. Papadrakakis M, Seismic assessment of structures and lifelines. Journal of Sound and Vibration, 334, 29-56, 2015.

[19] M.S. Saiidi, A. Arêde, D. Cardone, P. Delgado, M. Dolce, M. Fischinger, T. Isaković, S. Pantazopoulou, G. Pekcan, R. Pinho and A. Sextos, Modelling of bridges for inelastic analysis. Geotechnical, Geological and Earthquake Engineering, 21, 5-84, 2012.

[20] R. Monteiro, Sampling based numerical seismic assessment of continuous span RC bridges. Engineering Structures, in-press, 2016.

[21] H. Crowley, R. Pinho, Period-height relationship for existing European reinforced concrete buildings. Journal of Earthquake Engineering, 8(1), 93-119, 2004.

[22] R.K. Goel, A.K. Chopra, Period Formulas for Moment-Resisting Frame Buildings. Journal of Structural Engineering, 123(11), 1454-1461, 1997.

[23] C. Bernier, R. Monteiro, P. Paultre, Using the Conditional Spectrum Method for Improved Fragility Assessment of Concrete Gravity Dams in Eastern Canada. Earthquake Spectra, In-Press, 2016. 
[24] C. Zelaschi, R. Monteiro, R. Pinho, Improved fragility functions for RC bridge populations. M. Papadrakakis, V. Papadopoulos, V. Plevris eds. 5th ECCOMAS Thematic Conference on Computational Methods in Structural Dynamics and Earthquake Engineering (COMPDYN 2015), Crete Island, Greece, 25-27 May, 2015.

[25] M.H. Kutner, C. Nachtsheim, J. Neter, Applied linear regression models. McGraw-Hill, Irwin, 2004.

[26] Gruppo di Lavoro MPS, Redazione della mappa di pericolosità sismica prevista dall'Ordinanza PCM 3274 del 20 marzo 2003. Rapporto Conclusivo per il Dipartimento della Protezione Civile, INGV, Milano-Roma, 2004. (in Italian).

[27] R.L. Iman, J.M. Davenport, D.K. Zeigler, Latin Hypercube Sampling (A Program User's Guide). Technical Report SAND79-1473, Sandia Laboratories, Albuquerque, 1980.

[28] A.M.J. Olsson, S.G. Sandberg, Latin hypercube sampling for stochastic finite element analysis. Journal of Engineering Mechanics, 128(1), 121-125, 2002.

[29] R. Monteiro, R. Delgado, R., Pinho, Using different uncertainty models for seismic assessment of RC bridges. COMPDYN 2015 - 5th ECCOMAS Thematic Conference on Computational Methods in Structural Dynamics and Earthquake Engineering, 887-899, Crete, May 25-27, 2015.

[30] S. Broglio, H. Crowley, R. Pinho, Structure-Dependent Displacement-Based Intensity Measure for Bridges. Proceedings of 14th European Conference on Earthquake Engineering and Engineering Seismology, Ohrid, Macedonia, 2010.

[31] OpenSees, Open System for Earthquake Engineering simulation. Pacific Earthquake Engineering Research Center, US, 2002. available from http://opensees.berkeley.edu/

[32] Matlab, version 7.1.0.246. Natick, Massachusetts: The MathWorks Inc., 2005. 